einstein

Publicação Oficial do Instituto Israelita

de Ensino e Pesquisa Albert Einstein

ISSN: 1679-4508 | e-ISSN: 2317-6385
Como citar este artigo:

Nobrega Pavão D, Buttignol M, Pereira AJ,

Tanjoni R, Almeida EH, Leisnock P, et al.

Eficiência no processo operacional: redução

dos lançamentos incorretos e garantia de

compliance na prestação de contas. einstein

(São Paulo). 2018;16(4):eGS4200. http://dx.doi. org/10.31744/einstein journal/2018GS4200

Autor correspondente:

Daniela Nobrega Pavão

Avenida Santa Catarina, 2.785 - Vila Santa Catarina CEP: 04378-500 - São Paulo, SP, Brasil

Tel.: (11) 2151-2063

E-mail: daniela.nobrega@einstein.br

Data de submissão:

6/9/2017

Data de aceite:

4/2/2018

Conflitos de interesse:

não há.

\section{Copyright 2018}

\section{(c) By}

Esta obra está licenciada sob

uma Licença Creative Commons

Atribuição 4.0 Internacional.
GESTÃO E ECONOMIA EM SAÚDE

Eficiência no processo operacional: redução dos lançamentos incorretos e garantia de compliance na prestação de contas

\section{Efficiency in the operational process: reduction of incorrect entries and guarantee of compliance in the rendering of accounts}

Daniela Nobrega Pavão', Monique Buttignol', Adriano José Pereira ${ }^{1,2}$, Renato Tanjoni', Ederson Haroldo Pereira de Almeida², Patricia Leisnock², Gabriela Sato' ${ }^{1}$ Eliézer Silva²

${ }^{1}$ Hospital Municipal Vila Santa Catarina, São Paulo, SP, Brasil.

${ }^{2}$ Sociedade Beneficente Israelita Brasileira Albert Einstein, São Paulo, SP, Brasil.

DOI: 10.31744/einstein_journal/2018GS4200

\section{RESUMO}

Objetivo: Verificar o impacto da metodologia Lean Seis Sigma na redução de lançamentos incorretos de receitas e despesas não apropriadas. Métodos: Processo de revisão e aplicação de metodologia Lean Seis Sigma, no período de dezembro de 2015 e setembro de 2016, em um hospital geral de alta complexidade da cidade de São Paulo (SP). Resultados: Foram auditados $3.756 .814(100 \%)$ lançamentos durante os meses de dezembro de 2015 e setembro de 2016. 0 nível Sigma foi demonstrado na evolução do processo e aumentou de 3,44 Sigma, em dezembro de 2015, para 5,92 Sigma, em setembro de 2016. Os lançamentos classificados como receitas e despesas não apropriadas chegaram a $0 \%$ ao término do estudo. Conclusão: 0 uso da metodologia Lean Seis Sigma foi eficiente na redução de lançamentos incorretos, na apuração correta de custos, na garantia de compliance na prestação de contas e na acurácia no projeto de custos e desfechos.

Descritores: Lean Six Sigma; Triple aim; Revisão de processos; Qualidade da assistência à saúde; Auditoria de custos; Eficiência profissional

\section{ABSTRACT}

Objective: To verify the impact of the Lean Six Sigma methodology in reducing incorrect entries of non-appropriated revenues and expenses. Methods: Process for the review and application of the Lean Six Sigma methodology between December 2015 and September 2016, in a high-complexity general hospital in the city of São Paulo (SP). Results: A total of 3,756,814 (100\%) entries were audited between December 2015 and September 2016. The Sigma level evolved over the course of the process and increased from 3.44 Sigma in December 2015 to 5.92 Sigma in September 2016. Entries classified as non-appropriated revenues and expenses were brought down to $0 \%$ at the end of the study. Conclusion: The use of the Lean Six Sigma methodology was efficient in reducing incorrect entries, calculating costs, ensuring compliance in rendering of accounts and accurately determining cost-outcome ratios.

Keywords: Lean Six Sigma; Triple aim; Process review; Quality of health care; Cost audit; Professional assessment

\section{INTRODUÇÃ̃o}

A busca pela eficiência operacional deve se iniciar pelo entendimento do processo de custeio das atividades assistenciais. A auditoria de contas verifica e 
contabiliza todos os procedimentos realizados com o paciente, como medicamentos, materiais e exames, conhecidos como lançamentos. Problemas nestes lançamentos geram a apuração incorreta dos custos, podendo levar a impactos financeiros e desperdícios de mão de obra.

Recentemente, o Institute for Healthcare Improvement (IHI), uma organização americana independente sem fins lucrativos, propôs o Triple Aim como um conjunto de objetivos estratégicos que visam aprimorar os sistemas de saúde, centrado em três dimensões: (1) melhorar a experiência do paciente em relação à assistência; (2) melhorar a saúde da população; e (3) reduzir o custo per capita dos cuidados de saúde. ${ }^{(1)} \mathrm{Na}$ maioria dos sistemas de saúde, estas três dimensões não se encontram integradas e nem são aplicadas.

O conceito do Triple Aim engloba ainda cinco componentes: foco no paciente indivíduo e na família; redesenho da estrutura e serviços primários de saúde; gestão da saúde da população; controle de custos; e integração e execução do sistema. ${ }^{(2,3)}$

Para se alcançarem metas de qualidade assistencial, atreladas a um dispêndio racional de recursos, muitas instituições, de forma complementar, utilizam a metodologia do Lean Seis Sigma, que provê técnicas e princípios de qualidade, visando ao desempenho praticamente sem erros. Isso porque o desempenho de uma empresa é medido pelo nível Sigma de seus processos, sendo aceitos três ou quatro níveis de desempenho Sigma como norma. As ferramentas são aplicadas com um modelo simples para melhoria de desempenho, conhecida como DMAIC, (definir-mensurar-analisar-melhorar-controlar - D: definir os objetivos da atividade de melhoria; M: medir o sistema existente; A: analisar o sistema para identificar maneiras de eliminar a distância entre o desempenho atual do sistema ou processo e objetivo desejado; M: melhorar o sistema; e C: controlar o novo sistema). ${ }^{(4)}$

Já a metodologia Lean Seis Sigma complementa o desenvolvimento de serviços em saúde e sintetiza esta abordagem, que leva a um programa integrado, gerando melhoria dos processos. Ainda, a Lean Seis Sigma incorpora ferramentas de diagnóstico e análise, resultando em boas práticas e soluções para os problemas que lidam com resíduos e consumo de tempo desnecessário. ${ }^{(5)}$ A integração do Seis Sigma ao Lean constitui estratégia abrangente, poderosa e eficaz para a solução de problemas e também para a criação de novos processos e produtos. ${ }^{(6)}$

Desta forma, a utilização de recursos da maneira mais racional possível, e a razão entre desfecho clínico e custo operacional a mais elevada possível tornam-se essenciais a um serviço de saúde, em que a excelência da qualidade assistencial é a meta. ${ }^{(7)}$ Assim, o entendimento da interação de diversas áreas é importante, para compreender os processos estabelecidos internamente. Este conhecimento propicia base para tomada de decisão e a melhora do desempenho do processo, com impactos relevantes e importantes nas atividades operacionais diárias. ${ }^{(8,9)}$ Pouco tem sido descrito na literatura sobre a eficiência no processo operacional na redução dos lançamentos incorretos, a qual pode contribuir para as especificidades da apuração de custos nos serviços de saúde. ${ }^{(10-13)}$

\section{OBJETIVO}

Verificar o impacto da metodologia Lean Seis Sigma na redução de lançamentos incorretos de receitas e despesas não apropriadas.

\section{| MÉTODOS}

Este estudo foi realizado em um hospital público municipal da cidade de São Paulo (SP), entre dezembro de 2015 e setembro de 2016, o qual faz parte de uma parceria entre o Hospital Israelita Albert Einstein e a Prefeitura da Cidade de São Paulo. O hospital iniciou suas atividades em junho de 2015.

Para fins de análise, foram incluídos apenas os lançamentos incorretos desse período, de todos os setores do hospital, sem exceção, com a finalidade de otimizar processos e reduzir custos, direcionando-os da maneira correta.

Para entender a lógica de operação do hospital estudado, no que se refere a custos, foram verificadas suas diversas áreas e avaliados seus pontos de melhoria, aplicando-se a Lean Seis Sigma, para otimizar o processo de lançamentos.

Para tal, a literatura sobre a metodologia Lean Seis Sigma foi revisada. Esta metodologia está estruturada para resolver problemas de melhoria contínua de processos, por meio do uso das diversas ferramentas específicas. $^{(4)}$

Para fins de análise, foram incluídos $100 \%$ dos lançamentos incorretos no período do estudo - e não apenas uma amostra -, de todos os setores do hospital, sem exceção. Lançamentos corretos não foram objeto do presente estudo.

Inicialmente foi elaborado o sumário do contrato do projeto, essencial para definições como objetivo, limites, indicador e benefício do trabalho. O detalhamento do processo ocorreu seguindo as fases do DMAIC, conforme demonstrado a seguir. 


\section{D - Fase de definição: análise da situação atual e mapeamento do fluxo de valor}

A definição se deu avaliando-se a necessidade do projeto; a formação do time; a demanda do cliente; a realização do processo no momento do estudo; o fluxo de materiais, insumos e fluxo de informações; e os desperdícios, bem como sua eliminação; e, consequentemente, construindo-se um novo fluxo de processo para um estado futuro. Foram definidos, então, a descrição do problema, o indicador do projeto, a meta, o escopo e os membros do time.

Para esta construção, foi utilizada a Matriz ARMI, que correspondeu ao tempo dispendido por cada membro da equipe para o projeto, definido de acordo com suas possibilidades. Também as necessidades foram interpretadas pelos indicadores Critical To Quality (CTQ), que correspondeu à visão do cliente, e Critical To Process (CTP), ou seja, a visão do negócio.

Nesta etapa, foi construído o SIPOC, suppliers (fornecedores), inputs (entradas), process (processo), outputs (saídas) e customer (clientes), buscando-se compreender quem eram os fornecedores, e quais eram os processos e os clientes de cada área.

Os CTQs do presente estudo foram: custo dos atendimentos realizados no hospital; entendimento de custo contra desfecho dos pacientes; caracterização do mecanismo dos diversos lançamentos; existência de itens sem apropriação correta, ou seja, receitas e despesas não apropriadas (RDNA).

Os CTPs foram não realizar as alterações em tempo hábil, antes do fechamento do mês; falta de atenção da equipe ao realizar os lançamentos, persistindo erros constantes; sempre existir lançamento a ser avaliado, persistindo as RDNA; conhecimento prévio de novos itens a serem lançados e sua parametrização.

Foi feito, ainda, o mapeamento de macroprocesso, que é o desenho do processo em forma de fluxograma, com a finalidade de facilitar seu entendimento. Este mapeamento foi feito no programa Bizagi Process Modeler e é apresentado na figura 1.

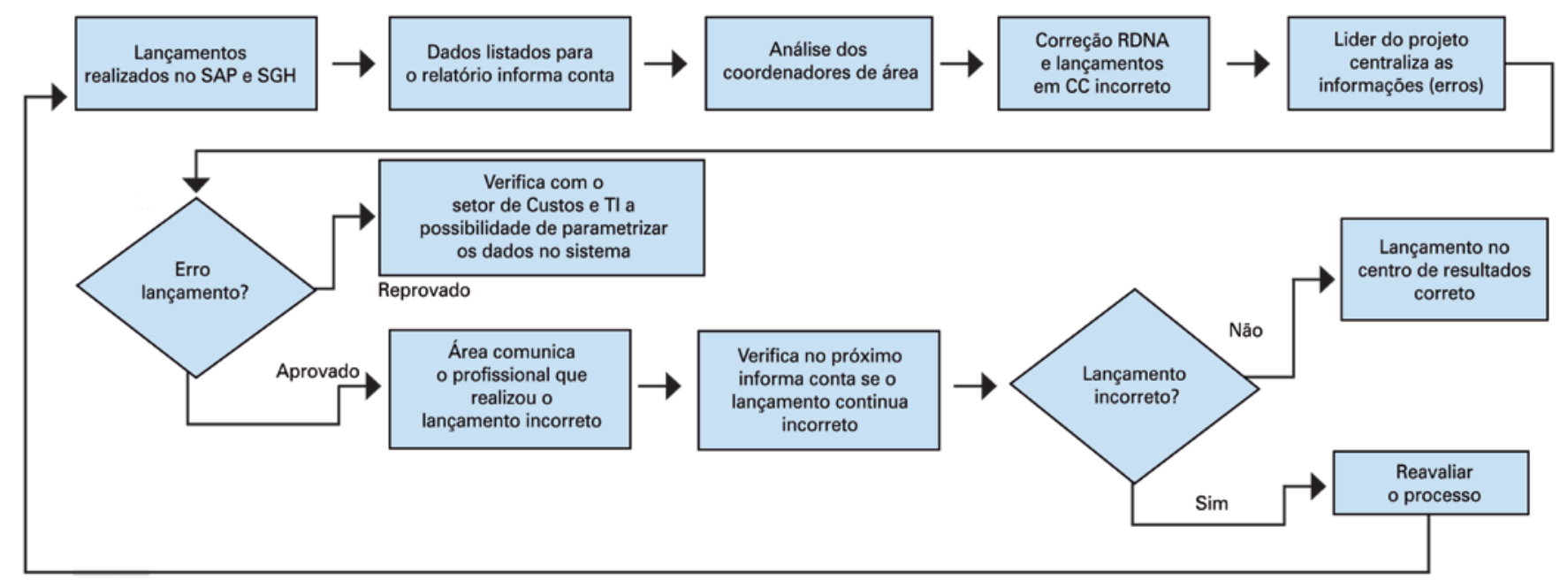

SAP: sistema, aplicativo e produtos para processamento de dados; SGH: sistema de gestão hospitalar; RDNA: receitas e despesas não apropriadas; CC: centro de custo; TI: Tecnologia da Informação.

Figura 1. Mapeamento de macroprocesso

M - Fase de mensuração e coleta dos dados: “Como o processo está hoje?" - Principais causas do problema Nesta fase, foi elaborado um mapa detalhado do processo. Nesse momento, para continuidade do trabalho, fez-se necessário o entendimento de todos os setores, que responderam se o fluxo do processo estava claro e se existia uma medição confiável. Além disso, uma coleta de dados sistematizada foi realizada a fim de se monitorarem a mensuração e os efeitos do plano de ação implementado (Figura 2).
Também foi aplicado o Diagrama Causa-Efeito, conhecido como Diagrama de Ishikawa, para possibilitar a visualização de todas as causas de um problema específico, por meio das principais entradas do processo (Figura 3).

Com relação à análise estatística, foram trabalhados $100 \%$ dos lançamentos, e a análise foi absoluta. A coleta de dados foi aplicada de forma planejada e validada em todos os itens, para confirmar a veracidade das informações. 


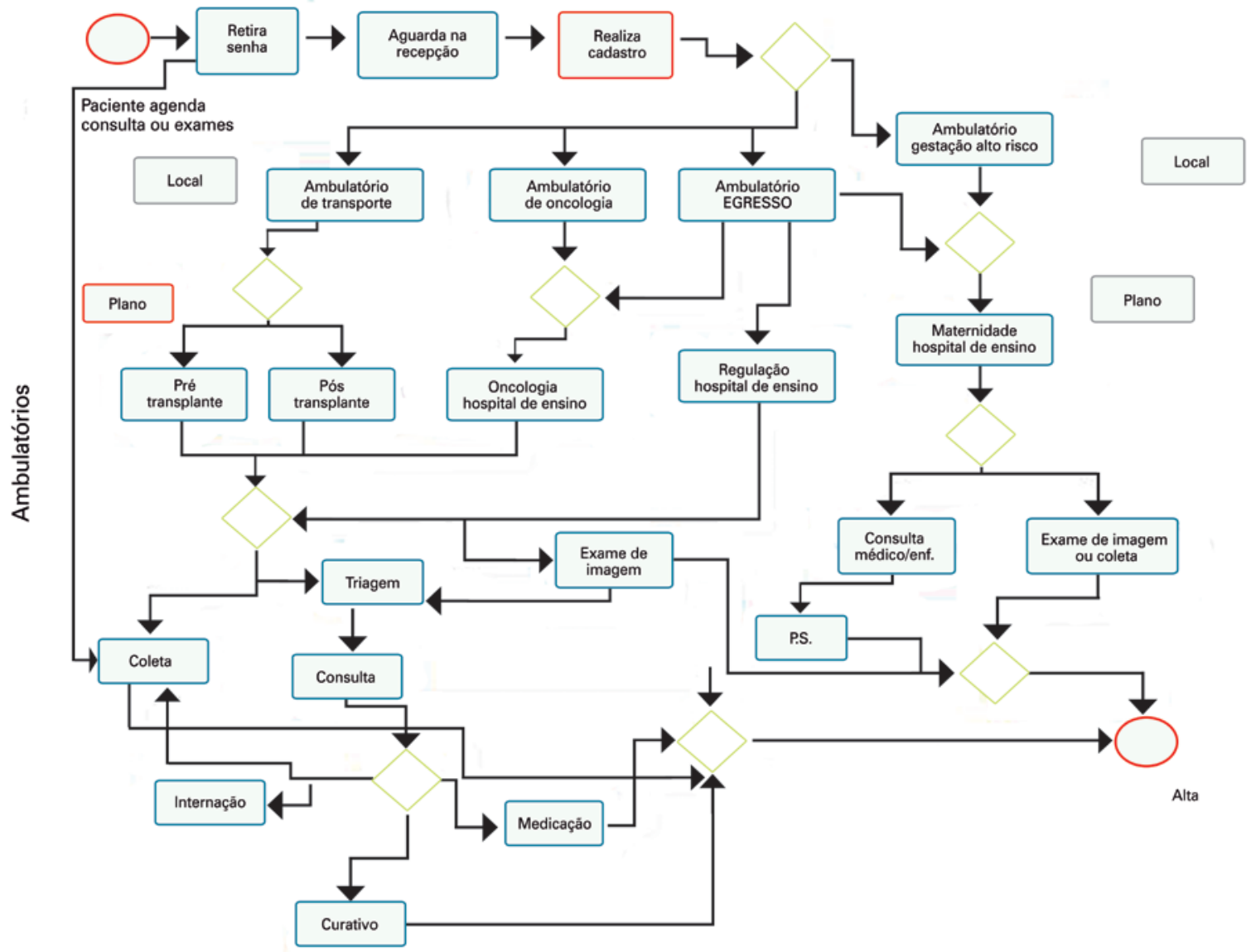

PS: pronto-socorro.

Figura 2. Exemplo de fluxograma criado para cada área. Neste caso, a figura representa o fluxograma do setor de ambulatório

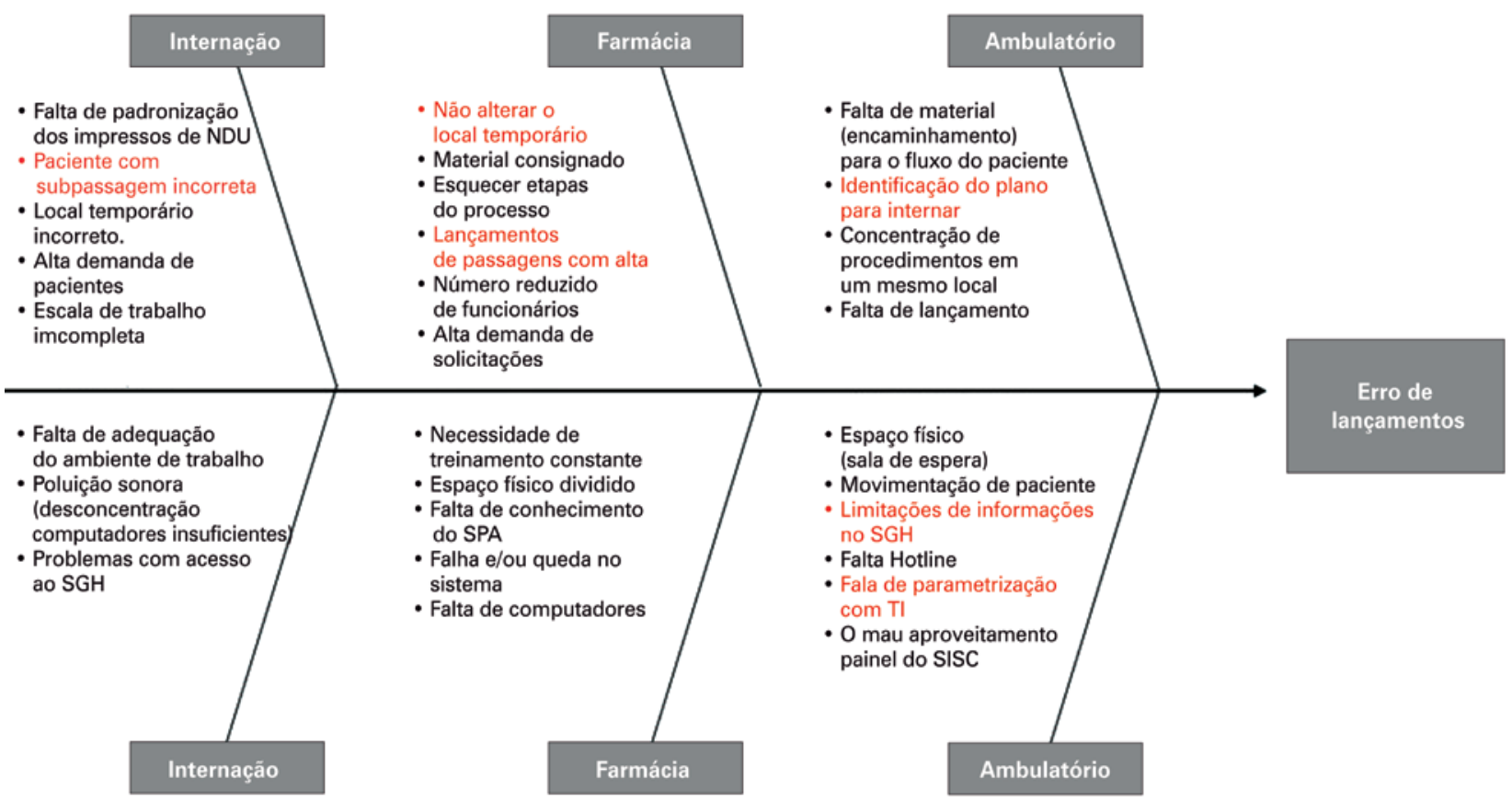

NDU: nota de débito unitário; SGH: sistema de gestão hospitalar; SAP: sistema, aplicativo e produtos para processamento de dados; TI: Tecnologia da Informação; SICS: sistema inteligente de chamada de senhas.

Figura 3. Diagrama Causa-Efeito que possibilita a visualização de todas as causas de um problema específico, considerando as principais entradas do processo 
A - Fase de análise: ferramentas para observar e analisar os dados coletados (análise de causa raiz, identificação de desperdício, e o que agrega

e não agrega valor)

Primeiramente, foi calculado nível Sigma inicial, que demonstra a proporção de erros. O nível Sigma 6 representa que, a cada 1 milhão de ocorrências, são encontradas 3,4 divergências (Figura 4).

\section{Nível Sigma}

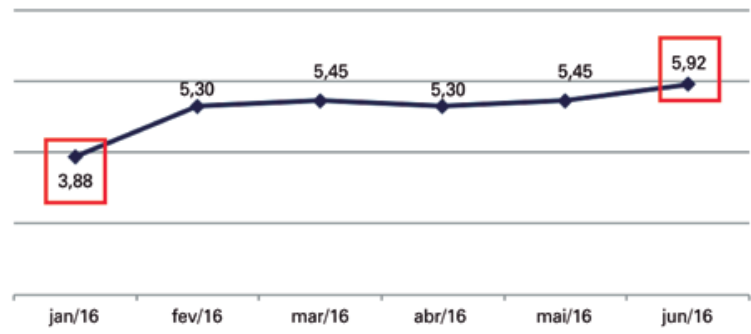

Figura 4. Apresentação da melhora do nível Sigma ao longo dos meses. Destaque para o mês de janeiro de 2016, com nível Sigma de 3,88, e 0 evolutivo até junho de 2016, cujo Sigma encontrado foi de 5,92
A aplicação do Diagrama de Pareto foi necessária, para analisar com detalhes os setores que mais apresentavam divergências nos lançamentos. Após as análises, foram definidas as metas, por meio do plano de ação. O ganho financeiro com o projeto foi calculado considerando a quantidade de erros prévios e seus respectivos valores no início do projeto, com a proporção da quantidade dos lançamentos atuais.

\section{I - Fase de melhoria: plano de ação e confirmação da otimização}

Nesta fase, ações simples, mas com grande impacto, foram realizadas para alcançar os objetivos definidos, diminuindo os erros de lançamentos. Uma destas ações foi a colocação de uma placa informativa com o local do centro de custo nos computadores em que eram realizados os lançamentos.

Após a validação de todos os processos, foi construído um fluxograma demonstrando a melhora do desempenho. (Figura 5).

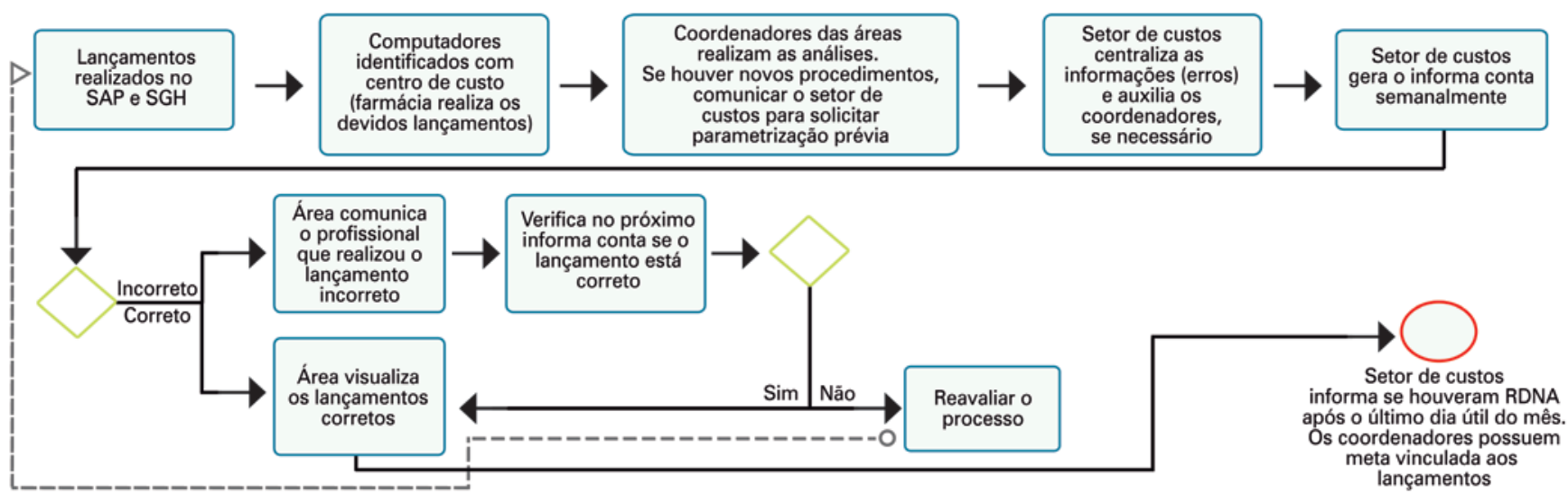

SGH: sistema de gestão hospitalar; SAP; sistema, aplicativo e produtos para processamento de dados, RDNA: receitas e despesas não apropriadas.

Figura 5. Fluxograma com o novo Mapa de Processo, desenhado a partir das ações de melhoria implementadas

\section{C - Fase de controle: controle estatístico do processo para uma metodologia à prova de erros}

O controle das soluções implementadas foi realizado mensalmente, com intuito de acompanhar os dados e auditar possíveis desvios. Esta fase contou com um novo cálculo do nível Sigma, um plano controle, a validação de resultados, a padronização do processo e a transição para o dono do processo.

\section{RESULTADOS}

De acordo com a tabela 1, observou-se a redução dos lançamentos incorretos durante a análise realizada en- tre os meses de dezembro de 2015 e setembro de 2016. Um total de 3.756 .814 (100\%) lançamentos foi auditado neste período. O nível Sigma foi demonstrado na evolução de 3,44 Sigma, em dezembro de 2015, para 5,92 Sigma, em setembro de 2016. O objetivo de $0 \%$ de RDNA foi atingido, conforme proposta inicial.

A tabela 2 apresenta a redução da perda de $\mathrm{R} \$ 1,8$ milhão quando comparadas as médias de dezembro de 2015 e janeiro de 2016 com os últimos meses do estudo (julho a setembro de 2016). Tal fato demonstra a importância da intervenção preventiva. Essa redução teve início em fevereiro de 2016, sendo o evolutivo do RDNA significativo, reduzindo bruscamente o valor de 1.803 
Tabela 1. Demonstração das receitas e despesas não apropriadas

\begin{tabular}{lcccc}
\hline Mês/ano & $\begin{array}{c}\text { Total de } \\
\text { lançamentos }\end{array}$ & RDNA & RDNA (R\$) & RDNA (\%) \\
\hline Dezembro/2015 & 170.754 & 1.262 & $51.171,00$ & 0,74 \\
\hline Janeiro/2016 & 207.490 & 1.803 & $71.630,00$ & 0,87 \\
\hline Fevereiro/2016 & 298.002 & 21 & $16.395,00$ & 0,01 \\
\hline Março/2016 & 401.875 & 19 & $4.763,00$ & 0,00 \\
\hline Abril/2016 & 510.118 & 37 & $2.807,00$ & 0,01 \\
\hline Maio/2016 & 332.153 & 19 & $4.130,00$ & 0,01 \\
\hline Junho/2016 & 399.299 & 2 & $2.065,00$ & 0 \\
\hline Julho/2016 & 459.897 & 20 & $7.354,00$ & 0 \\
\hline Agosto/2016 & 489.037 & 7 & $8.273,00$ & 0 \\
\hline Setembro/2016 & 488.189 & 9 & $2.792,00$ & 0 \\
\hline
\end{tabular}

Tabela 2. Cálculo de redução de perda

\begin{tabular}{|c|c|c|c|c|c|}
\hline & \multirow{2}{*}{$\begin{array}{c}\text { Antes } \\
\text { (dezembro/2015- } \\
\text { janeiro/2016) }\end{array}$} & \multirow{2}{*}{$\begin{array}{c}\text { Depois } \\
\text { (julho/2016- } \\
\text { setembro/2016) }\end{array}$} & \multicolumn{3}{|c|}{ Redução de perda } \\
\hline & & & $\begin{array}{c}\text { Por } \\
\text { lançamento }\end{array}$ & Mensal & Anual \\
\hline $\begin{array}{l}\text { RDNA/ } \\
\text { lançamento } \\
\text { (R\$) }\end{array}$ & 0,32 & 0,01 & 0,312 & 149.386 & 1.792 .634 \\
\hline
\end{tabular}

(janeiro/2016) para 21 (fevereiro/2016). Nos meses subsequentes, houve continuidade desta redução significativa das perdas.

Entre os meses de fevereiro e junho de 2016, foi realizado um levantamento para identificação dos problemas, tendo sido identificados como principais ocorrências de erro o lançamento sem parametrização $(33 \%)$ e o lançamento em centro de custo incorreto, pelo fato de a farmácia atender várias unidades (30\%).

\section{| DISCUSSÃO}

O principal achado deste estudo foi o fato de a metodologia Lean Seis Sigma, por meio de seus processos, auxiliar na diminuição dos lançamentos incorretos observados na auditoria de contas, bem como todos os procedimentos relacionados à internação do paciente, como medicamentos, materiais e exames. Os processos envolvidos na auditoria de contas estão diretamente relacionados à eficiência na redução de lançamentos incorretos, apuração correta de custos, garantia de compliance na prestação de contas e acurácia no projeto de custos e desfechos, pois visam à eficiência do processo operacional na apuração dos resultados financeiros. Assim, a aplicação de uma ferramenta capaz de identificar e gerar melhoria é necessária nos serviços de saúde.
Para melhorar o processo de lançamento, foram dadas as devidas explicações aos coordenadores das áreas, abordando desde como realizar os lançamentos até o impacto do erro. Os erros que aconteceram com mais impacto foram os itens que não estavam padronizados no sistema, tendo sido feito o ajuste deles. Para tal, foi estabelecida uma parceria com o setor da Tecnologia da Informação.

Muitos processos também eram realizados indevidamente. Para se conhecer este fato, foram mapeados todos os setores, principalmente aqueles que ofereciam mais oportunidade de erros, como ambulatório, pronto-socorro da ginecologia, clínica médico-cirúrgica, materno-infantil, e farmácia.

Ainda, algumas estratégias simples foram utilizadas para diminuir o desperdício de tempo, gerando resultados significativos. Por exemplo: alguns lançamentos eram realizados em centro de custo incorreto, como centro cirúrgico e obstétrico, considerados locais temporários. Assim, foi feito o ajuste manual do centro de custo em cada lançamento incorreto realizado no sistema.

A integração entre o Lean e o Seis Sigma é cada vez mais evidente. ${ }^{(14)}$ Em termos conceituais, a ideia é tratar o Lean com foco principalmente na eliminação dos desperdícios e no aumento da velocidade dos processos, ${ }^{(15)}$ e o Seis Sigma pode reduzir a variabilidade - e, consequentemente, os defeitos. $^{(4)}$

A gestão Lean não é um conceito novo, mas sua implementação nos cuidados de saúde é relativamente nova. ${ }^{(16)}$ Muitos aspectos do Sistema Toyota de Produção e de outras ferramentas podem se aplicar aos processos de prestação de cuidados de saúde. ${ }^{(16-19)}$ Algumas organizações de saúde com visão de futuro, como o Virginia Mason Medical Center (Seattle, W Seattle, Washington, USA), lideram atualmente o caminho, demonstrando que este estilo de gestão pode reduzir o desperdício em cuidados de saúde, oferecendo resultados comparáveis aos de outras indústrias. ${ }^{(20)}$ Desse modo, nosso serviço vem trilhando por este mesmo caminho.

Em adição, a aplicação dessa ferramenta evidencia que os fatores mais importantes se relacionavam aos lançamentos realizados incorretamente, ocorrendo a alocação no centro de custo transitório de RDNA nas diversas passagens dos pacientes do hospital. Foi, então, necessário acompanhar, de forma efetiva e precisa, os motivos destes erros. Antevia-se, na oportunidade, o aumento do número de passagens atendidas mensalmente, conforme novos setores tornavam-se operacionais.

Além da RDNA, lançamentos aconteciam de forma incorreta nos diversos centros de custos, o que demandou a apropriação correta dos lançamentos indevidos em sua totalidade, para minimizar o impacto na análise entre custo e desfecho do paciente. 
Para a eficaz implementação do Lean Seis Sigma, faz-se necessária uma mudança cultural, que somente pode ocorrer com o comprometimento da alta liderança. Em nosso estudo, a liderança exerceu este papel fundamental, ao acompanhar, envolver e conscientizar toda equipe, por meio de estratégias como reuniões semanais com coordenadores de área, para expor resultados e atribuir metas - esta foi a medida mais eficaz observada.

Nosso hospital inspira-se conceito do Triple Aim, que descreve, em sua abordagem, a otimização do desempenho do sistema de saúde, visando que novos projetos seja desenvolvidos para buscar simultaneamente suas três dimensões: melhorar a experiência do paciente no cuidado (incluindo qualidade e satisfação); melhorar a saúde das populações; e reduzir o custo per capita dos cuidados de saúde.(2)

Ao realizar uma associação entre a Lean Seis Sigma e o Triple Aim, pode-se afirmar que ambos:

- Melhoram a experiência do paciente em relação à assistência: com a redução do retrabalho, o desperdício de tempo foi reduzido, e o tempo dedicado ao paciente aumentou. É possível melhorar a experiência do paciente por meio dos relatos nos conselhos consultivos (encontro entre a liderança, a equipe, os pacientes e os familiares) realizados bimestralmente em cada área.

- Melhoram a saúde das populações: com a otimização dos processos de lançamentos, a alocação do custo é realizada de maneira eficaz, auxiliando no orçamento efetivo do serviço, melhorando seu planejamento e, consequentemente, a apuração dos resultados sem variabilidade. Há, assim, melhora dos recursos financeiros aplicados à população.

- Reduzem o custo per capita dos cuidados de saúde: a apropriação correta dos lançamentos indevidos contribui para o projeto custo e desfecho do Programa de Desenvolvimento Institucional do Sistema Único de Saúde (PROADI-SUS), uma ação do Ministério da Saúde dirigida ao fortalecimento do SUS para parceria com hospitais filantrópicos de qualidade reconhecida. ${ }^{(3,21)}$

Sem uma cultura receptiva, os princípios do Lean Seis Sigma não seriam possíveis. O IHI acredita que muitas ferramentas de gerenciamento e operações de outras indústrias podem ser aplicadas com sucesso aos cuidados de saúde. A promessa de reduzir ou eliminar desperdício de tempo, dinheiro e energia em cuidados de saúde, e a criação de um sistema eficiente, eficaz e verdadeiramente sensível às necessidades dos pacientes (os “clientes") são fundamentais e culminam com os princípios do projeto Lean Seis Sigma. ${ }^{(20)}$
Em nosso estudo realizamos medição, notificação e comparação dos resultados, as quais parecem ser atualmente as melhores maneiras de se implementar o valor baseado no cuidados de saúde, melhorando os resultados e fazendo boas escolhas em relação ao custo desfecho de cada paciente. ${ }^{(22,23)}$

A principal limitação do presente estudo foi o sistema integrado utilizado no serviço para a realização dos lançamentos. Algumas melhorias observadas não puderam ser aplicas por conta da restrição deste sistema.

Conseguir fazer todas estas ações sem aumento do número de funcionários ou de equipe dedicada exclusivamente ao projeto foi um desafio. Para alcançar o resultado, foram necessários organizar o processo e saber priorizar as situações imprevistas, que surgiram durante o processo. Ao final, não houve gasto adicional em nenhuma linha de custos; os recursos foram reaproveitados e redesenhados para alcance dos resultados; e uma nova cultura foi implementada em nosso serviço. A transição foi realizada para o setor de custos, que deve acompanhar e realizar as devidas auditorias, dando continuidade à melhoria implementada. Não foram realizados testes estatísticos neste estudo; a análise dos lançamentos foi absoluta, ou seja, todos os lançamentos foram auditados, analisados e corrigidos, chegando o número de lançamentos incorretos a próximo de zero. Portanto, não houve análise estatística para avaliar diferença estatisticamente significante, e sim a melhora importante no processo por meio dos resultados.

\section{CONCLUSÃO}

As metodologias Lean Seis Sigma foram eficazes na redução dos lançamentos incorretos alocados como receitas e despesas não apropriadas, na apuração correta de custos, na garantia de compliance na prestação de contas, e na acurácia no projeto de custos e desfechos. A meta proposta de redução foi atingida conforme proposta inicial, demonstrando a melhoria dos processos no lançamento. Quanto mais próximo o nível Sigma de Seis, melhor a excelência do resultado, o que demonstra a confiabilidade dos dados para demonstrar o custo e os desfecho dos pacientes no hospital estudado.

\section{AGRADECIMENTOS}

À equipe do Lean Seis Sigma do Hospital Vila Santa Catarina: Priscilla Santini Ramalho, Patricia Helena Moreira da Silva, Klívia Neri, Marco Antonio de Salvo Júnior, Valéria Rafael da Silva, Michele Sevarolli, Bruna de Assis, Thiago Pocheca Mattos e José Marcos Batista da Silva. 


\section{IINFORMAÇÕES DOS AUTORES}

Nobrega Pavão D: https://orcid.org/0000-0002-4905-3045

Buttignol M: https://orcid.org/0000-0002-7195-6400

Pereira AJ: https://orcid.org/0000-0002-9467-6516

Tanjoni R: https://orcid.org/0000-0002-5150-2903

Almeida EH: https://orcid.org/0000-0002-3903-4465

Leisnock P: https://orcid.org/0000-0002-4555-6526

Sato G: https://orcid.org/0000-0002-0417-6615

Silva E: https://orcid.org/0000-0002-0725-7832

\section{REFERÊNCIAS}

1. Berwick DM, Nolan TW, Whittington J. The triple aim: care, health, and cost. Health Aff (Millwood). 2008;27(3):759-69.

2. Institute for Healthcare Improvement (IHI). Triple Aim - The Best Care for the Whole Population at the Lowest Cost [Internet]. Boston: IHI; 2018 [cited 2017 June 22]. Available from: http://www.ihi.org/Engage/Initiatives/ TripleAim/Pages/default.aspx

3. Bisognano M, Keeney C. Buscando o Triple Aim na Saúde: 8 inovadores mostram o caminho para melhor tratamento, melhor saúde e menos custos. São Paulo: Atheneu; 2015.

4. Pyzdek T, Keller P. Seis Sigma: guia do profissional- um guia completo para green belts, black belts e gerentes de todos os níveis. Rio de Janeiro: Alta Books; 2011. 560 p.

5. de Koning H, Verver JP, van den Heuvel J, Bisgaard S, Does RJ. Lean Six Sigma in healthcare. J Healthc Qual. 2006;28(2):4-11.

6. Hors C, Goldberg AC, Almeida EH, Babio Júnior FG, Rizzo LV. Application of the enterprise management tools Lean Six Sigma and PMBOK in developing a program of research management. einstein (São Paulo). 2012;10(4):480-90.

7. Does RJ, van den Heuvel J, Mast J, Niemeijer GC. Improving quality in healthcare while reducing costs. Quality Management Forum. 2010;36(3):12-5.

8. Langley GJ, Moen RD, Nolan KM, Nolan TW, Norman CL, Provost LP. Modelo de melhoria: uma abordagem prática para melhorar o desempenho organizacional. São Paulo: Mercado de Letras; 2011.

9. Kilo CM. A framework for collaborative improvement: lessons from the Institute for Healthcare Improvement's Breakthrough Series. Qual Manag Health Care. 1998;6(4):1-13.

10. Bonafide CP, Localio AR, Song L, Roberts KE, Nadkarni VM, Priestley M, et al. Cost-benefit analysis of a medical emergency team in a children's hospital. Pediatrics. 2014;134(2):235-41.
11. Naughton BJ, Moran MB, Feinglass J, Falconer J, Williams ME. Reducing hospital costs for the geriatric patient admitted from the emergency department: a randomized trial. J Am Geriatr Soc. 1994;42(10):1045-9.

12. Dimick JB, Chen SL, Taheri PA, Henderson WG, Khuri SF, Campbell DA Jr. Hospital costs associated with surgical complications: a report from the private-sector National Surgical Quality Improvement Program. J Am Coll Surg. 2004;199(4):531-7.

13. Lopes LA, Dyniewicz AM, Kalinowski LC. [Material management and hospital cost in neonatal ICU]. Cogitare Enferm. 2010;15(2):278-85. Portuguese.

14. Snee RD. Lean Six Sigma: getting better all the time. Int J Lean Six Sigma. 2010;1(1):9-29.

15. Joosten T, Bongers I, Janssen R. Application of lean thinking to health care: issues and observations. Inter J Qual Health Care. 2009;21(5):341-7.

16. Teich ST, Faddoul FF. Lean management: the journey from toyota to healthcare. Rambam Maimonides Med J. 2013;4(2):e0007.

17. Gijo EV, Antony J. Reducing patient waiting time in outpatient department using Lean Six Sigma methodology. Qual Reliab Engng Int. 2014,30:1481-91. https://doi.org/10.1002/qre.1552

18. Improta G, Balato G, Romano M, Carpentieri F, Bifulco P, Alessandro Russo $M$, et al. Lean Six Sigma: a new approach to the management of patients undergoing prosthetic hip replacement surgery. J Eval Clin Pract. 2015; $21(4): 662-72$.

19. Bandyopadhyay JK, Coppens K. Six Sigma Approach to Healthcare Quality and Productivity Management. Inter J Quality Productivity Manag. 2005;5(1):1-12.

20. Institute for Healthcare Improvement $(|\mathrm{H}|)$. Going Lean in Health Care [Internet]. Cambridge, MA: IHI; 2005 [Innovation Series 2005]. [cited 2017 Jun 22]. Available from: https://www.entnet.org/sites/default/files/ GoingLeaninHealthCareWhitePaper-3.pdf

21. Brasil. Ministerio da Saúde. Departamento de Ciência e Tecnologia, Secretaria de Ciência, Tecnologia e Insumos Estratégicos. Programa de Desenvolvimento Institucional do Sistema Único de Saúde. Rev Saúde Pública. 2011;45(4):808-11.

22. Freitas R, Fukomoto HL. Implantação do custeio baseado em atividades ABC/ ABM no setor hospitalar: centro cirúrgico, um caso prático. In: VIII Congresso Brasileiro de Custos [Internet]. 2001; São Leopoldo, 3-5 Out [citado 2018 Jan 30]. Disponível em: https://anaiscbc.emnuvens.com.br/anais/article/ view/2817/2817

23. Pereira AJ, Ferraz LJ, Sato G, Kanamura AH, Tanjoni R, Neves HS, et al. Valuebased health care in a public hospital in Brazil. Review Pub Adm Manag. 2015;3(1):1-2. 